\title{
A C-Band Planar Metamaterial-Inspired Antenna Employing a Hexagonal Cell Periodic Structure
}

\author{
Humberto X. de Araujo, Mauricio W. B. Silva, Ivan R. S. Casella, Antonio L. P. S. Campos, and \\ Carlos E. Capovilla
}

\begin{abstract}
In this paper, a metamaterial inspired antenna to operate in the C-band is designed, fabricated, and characterized. The structure is based on the metamaterial tripolar array, to obtain a better gain, bandwidth, and frequency resonance when compared to a conventional circular UWB antenna with same structural characteristics. Simulation and experimental results show an increase of about $32 \%$ on the bandwidth and $9 \%$ on the gain with a satisfactory radiation pattern.
\end{abstract}

Index Terms-UWB, patch antennas, metamaterial, FSS.

\section{INTRODUCTION}

A wideband antenna is usually applied in wireless systems with high data transmission, like radar, satellite, medical, and multimedia in general [1-4].

In recent years, planar antenna becomes a crucial element to the RF transceivers, due to its satisfactory performance with a simple fabrication, compact design, and normally, low cost. Its easily adapt to portable and mobile devices, in addition of these good characteristics, provide the versatility needed to the modern communication system.

However, the bandwidth is a serious deficiency of resonant planar antennas, with values about 2\% [5-8]. Among them, patch antennas also present low gain, which compromises its use in several applications. To overcome these problems, the metamaterial technology can be applied to planar antennas to improve its overall behavior, and eventually, achieve the best performance [9-10]. In this way, the application of metamaterial technology, as Frequency Selective Surfaces (FSS), on patch antennas, can result in an increase of its low gain and bandwidth [11-12].

In fact, metamaterials are formed by unitary cells, periodically organized, with the objective of provide negative

H. X. Araujo is with the Federal University of Tocantins, Palmas, Tocantins Brazil (e-mail: hxaraujo@uft.edu.br).

M. W. B. Silva is with Federal University of Roraima, Boa Vista, Roraima, Brazil (e-mail: mauricio.weber@gmail.com).

I. R. S. Casella is with Federal Univeristy of ABC, Santo André, Brazil (email: icasella@gmail.com).

A. L. P. S. Campos, is with Federal University of Rio Grande do Norte, Natal, Brazil (e-mail: alpscampos@gmail.com).

C. E. Capovilla is with Federal Univeristy of ABC, Santo André, Brazil (email: carlos.capovilla@ufabc.edu.br).

Digital Object Identifier: 10.14209/jcis.2018.4 values of $\mu$ and $\varepsilon$ [13-14]. The negative refraction is one of the properties of the metamaterials. This one is possible due to its electric permittivity and magnetic permeability, $\varepsilon$ and $\mu$, to achieve negative values.

In this context, a C-band (4-8GHz) Metamaterial-Inspired antenna employing Tripolar Array unitary cell (TAMI antenna) was designed with a proposal of improving its response in terms of bandwidth and gain keeping good radiation pattern characteristics. Several simulated and measured results are present with satisfactory results.

Besides this introductory section, the paper is organized as follows: FSS theory is present in Section 2; the proposed antenna and its design are shown in Section 3; the main results are considered in Section 4; and, Section 5 concludes the work.

\section{FSS THEORY: TRIPOLAR ARRAY UNITARY CELL}

The use of the correct modeling technique can define the results accuracy in the analysis of FSS. Classical numerical methods, such as Finite Difference Time Domain (FDTD) and Finite Element Method (FEM) produce accurate results at cost of higher computational effort and processing time [15]. Hybrid methods can be used to partially overcome problems presented by FDTD and FEM methods. Integral Equation Method (IEM) is one of the most efficient. It can not be solved by closed-form equations, but must addressed by numerical techniques, such as Method of the Moments (MoM) [16]. The equivalent circuit model is one of the simplest methods. It is a less accurate technique but requires very limited computational resources. In this analysis, various printed segments are modeled as inductive and capacitive elements which are connected by transmission lines. From the solution of this circuit the transmission and reflection FSS characteristics are found [17-18].

In [19] the authors merged the equivalent circuit model and MoM to obtain the Green's functions and analyze periodic structures. The method is simple and shows accurate results. In this work, a similar analysis method based on the proposed model in [19] is used as an initial procedure to the proposed antenna design. The method uses a circuit approach in conjunction with the transmission lines model for analyzing High Impedance Surfaces (HIS) and is subsequently applied to design an antenna in order to improve its performance. Although the approach presented in [11] is more appropriate to the problem of the tripolar array unit cell then the one 
proposed here, the obtained results showed good agreement with numerical results, and feasible, so for this application.

This analysis is dedicated to the modeling of FSS, which are the key element of HIS structures in the presence of the dielectric substrate. In this configuration additional transmission lines representing dielectric layers, as illustrated in Figure 1, must be considered in the extraction of the constituent parameters such as $L$ and $C$. The initial modeling for the concentrated parameters extraction in the freestanding configuration was presented in [20] and will not be considered again. Considering the FSS between dielectric layers and leaving the freestanding configuration, presented in [21-22] the input impedance of the structure can be obtained by classical transmission line equations (see Eq.1). Thus, the impedance of the FSS is obtained by solving a simple parallel circuit.

$$
\begin{gathered}
Z_{\text {ent }}=Z_{1} \frac{1+\Gamma i n}{1-\Gamma i n} \\
\Gamma_{e n t}=\frac{Z_{0}\left(1+\Gamma_{d}\right)-Z_{1}\left(1-\Gamma_{d}\right)}{Z_{0}\left(1+\Gamma_{d}\right)+Z_{1}\left(1-\Gamma_{d}\right)} e^{j 2 \beta_{1} d}
\end{gathered}
$$

where: $Z_{1}$ is the impedance of the first dielectric layer, $\beta_{1}$ is the phase constant of the first dielectric layer, $d$ is the thickness of the first dielectric layer, $\Gamma_{d}$ represents the input reflection coefficient computed by the MoM analysis, and $\Gamma_{\text {in }}$ is the input reflection coefficient.

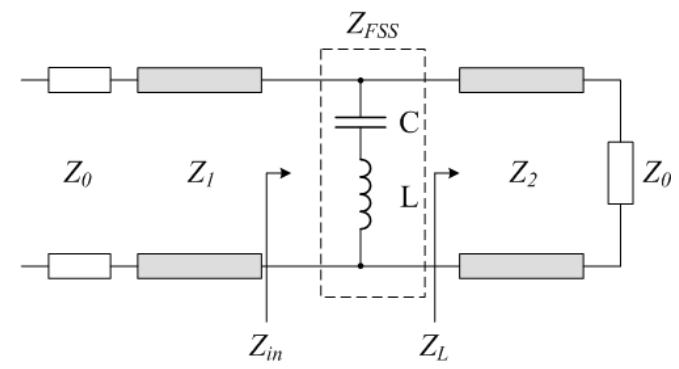

Fig. 1. Equivalent circuit model for FSS between dielectric layers.

The capacitance in function of the thickness and permittivity of the dielectric substrate was analyzed through simulations. For it, the method presented in [13] was used, however, with the value of impedance corrected for FSS between dielectrics. Thus, the optimal capacitances are computed and normalized to freestanding value in order to highlight the effect of the dielectric.

The normalized capacitance approximates the value of dielectric permittivity as the substrate thickness approaching the value $\mathrm{D} / 2$, where $\mathrm{D}$ is the periodicity of the unitary cells.

It is important to note that this study aimed to analyze the effect of the dielectric on the structure behavior, which results in a negligible effect on the inductance. For this reason, inductance is not performed at this analysis.

Using Eq. 3 [17], it was possible to perform the adjustment of the dependence of the effective permittivity of thickness by factor $N$, which is an exponential factor that takes into account the slope of the curve.

$$
\varepsilon_{\text {eff }}=\varepsilon_{r}+\left(\varepsilon_{r}-1\right)\left(\frac{-1}{e^{N x}}\right)
$$

In this Figure 2 is presented the comparison of the analytical data and results obtained from the regression model, in which $\mathrm{N}$ was obtained for $\varepsilon \mathrm{r}=3.55$. To the structure used in this study, the best fit value was $N=0.63$.

In practical designs, it is needed to use substrates with different thicknesses and/or permittivity. One reason is the improvement in the angular stability obtained when dielectric layers are used.

The analysis of the parameters not only considers a structure that encompasses a greater amount of physical parameters, but also shows the model application viability in more complex structures. At the same model, any geometry can be considered in the presence of dielectric substrates, and for normal and oblique incidences.

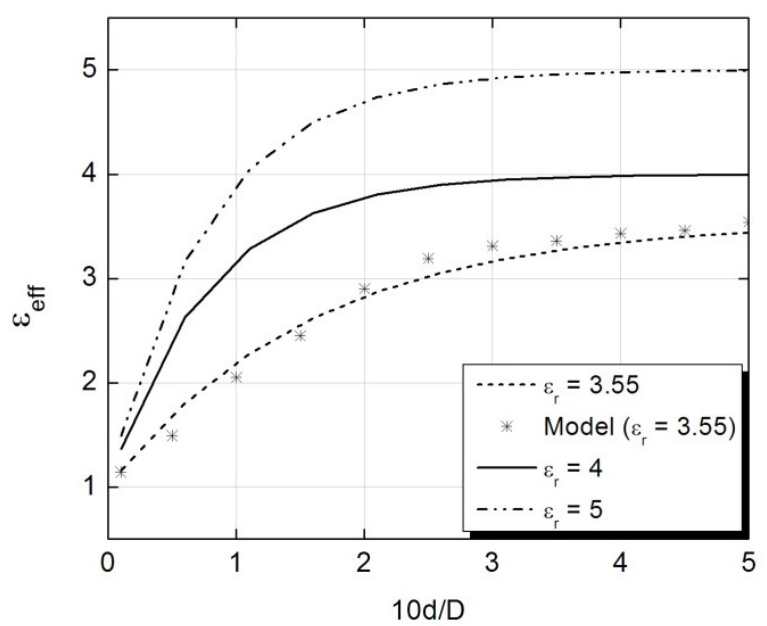

Fig. 2. Variation of the effective dielectric constant depending on the thickness of the substrate to the FSS between dielectrics.

The analysis of the proposed structure is based on the equivalent circuit model together with the transmission line modeling method, which was presented in [20]. The presented method can be applied in the analysis of any type of FSS element and offers a fast and precise analysis, when compared to other methods. Figure 3 shows the FSS embedded between two dielectric layers with both different thickness and dielectric permittivity .

In this way, the FSS is considered to be embedded between two dielectric substrates with different thickness and dielectric constant, and under both normal and oblique incidence. According to the classical equations of transmission lines and considering the equivalent substrates such as transmission lines, as well as, the analysis of the effect of the dielectric, the impedances across the plates can be obtained as [19]:

$$
Z_{E}^{T E, T M}=Z_{m}^{T E, T M}\left[\frac{\left[Z_{L}^{T E, T M}+Z_{m}^{T E, T M} \tanh \left(j \beta_{m} d\right)\right.}{Z_{m}^{T E, T M}+Z_{L}^{T E, T M} \tanh \left(j \beta_{m} d\right)}\right]_{(4)}
$$

where: $Z_{L}$ is the load impedance. The $Z_{m}{ }^{T E}, Z_{m}{ }^{T M}$ and $\beta_{m}$ terms are, respectively, the characteristics impedances of the plate 
for $T E$ and $T M$ modes, and the propagation constant along the normal drive plate. These terms are given by:

$$
\begin{aligned}
& Z_{m}^{T E}=\frac{\omega \mu}{\beta_{m}}, Z_{m}^{T M}=\frac{\beta_{m}}{\omega \varepsilon} \\
& \beta_{m}=\sqrt{k_{m}^{2}-k_{t}^{2}}, k_{t}=k_{0} \operatorname{sen}(\theta), k_{m}=k_{0} \sqrt{\varepsilon_{r} \mu_{r}}
\end{aligned}
$$

where: $k$ is a number of transverse waves, $\theta$ the incidence angle, and $k_{0}$ the propagation constant in free space.

As an initial analysis, for normal incidence of the plane wave, an array of tripole [11] is considered between two dielectric substrates with different thicknesses and permittivity. The parameters of the geometry are: $p=10 \mathrm{~mm}$, $\mathrm{w}=1 \mathrm{~mm}$, and $1=8.7 \mathrm{~mm}$, while the parameters of the substrates are: $\varepsilon r 1=3, \varepsilon r 2=5, \mathrm{~d} 1=1 \mathrm{~mm}$ and $\mathrm{d} 2=2 \mathrm{~mm}$. These values are used for the first approximation and analysis.

The results of the model and the electromagnetic simulation analysis, as shown in Figure 3, are in good agreement for the order Floquet propagating modes. The proposed method is valid until the first resonance. After it, the method does not consider the physical parameters and loses its validity, in this way, the presented simulation was realized up to $5 \mathrm{GHz}$ in Figures 3 and 4.

To investigate the behavior under oblique incidence, remained the tripoles arrangement embedded between dielectric plates with different thickness and permittivity, a wave with the angle out of the normal was considered. Figure 4 shows the validation model for oblique incidence of a TE wave. In this case, a wave focusing to $60^{\circ}$ was considered. Again, it is observed a good agreement of the model to the structure up to the first resonance.

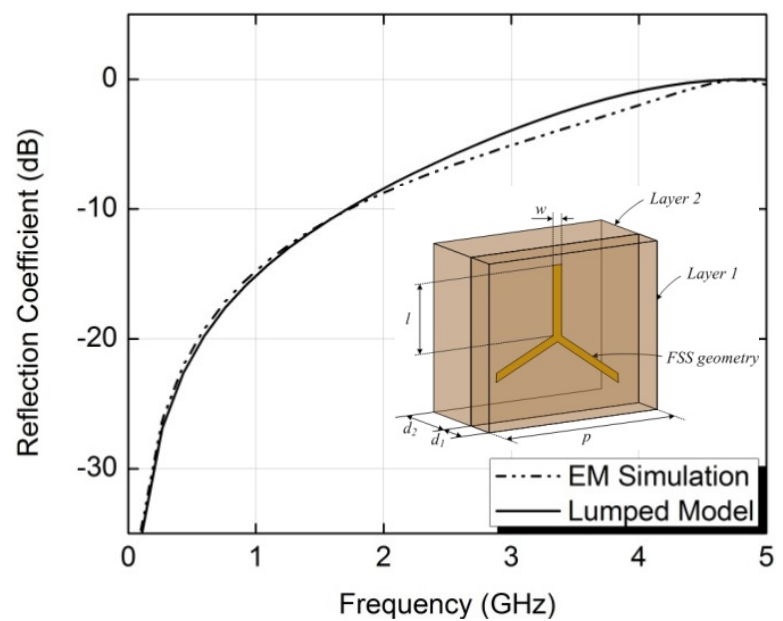

Fig. 3. Comparison between the reflection coefficient of an embedded FSS obtained by the EM simulation and the lumped model.

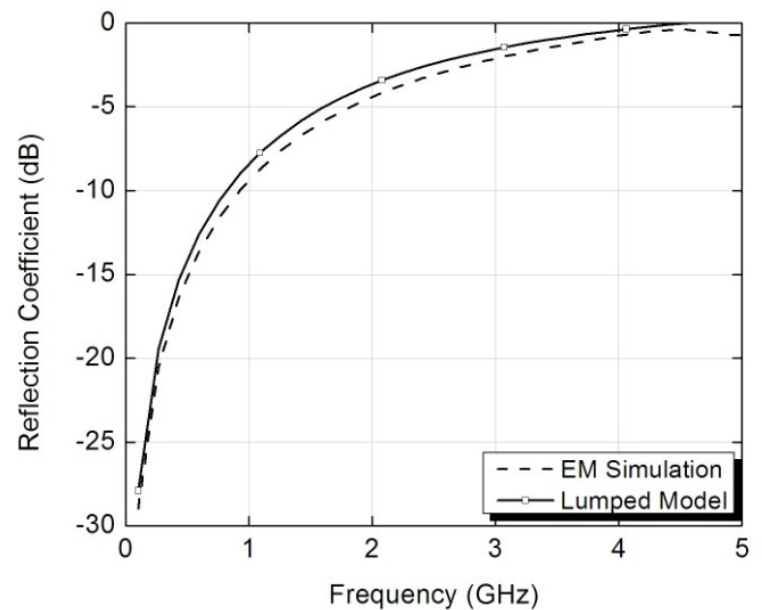

Fig. 4. Comparison Validation of the model for oblique $\left(60^{\circ}\right)$ incidence of a TE wave.

Define abbreviations and acronyms the first time they are In the Figure 5 is presented the results for normal and oblique incidence $\left(60^{\circ}\right)$. This figure shows the behavior of the tripolar array unitary cell that is used in the proposed UWB antenna in all C-Band range. This analysis through EM simulation shows the satisfactory and adequate behavior of the cell, instigating its utilization on the design of a modern planar antenna.

With the study carried out, the model was used for the design a structure in order to improve the performance of a circular UWB patch antenna.

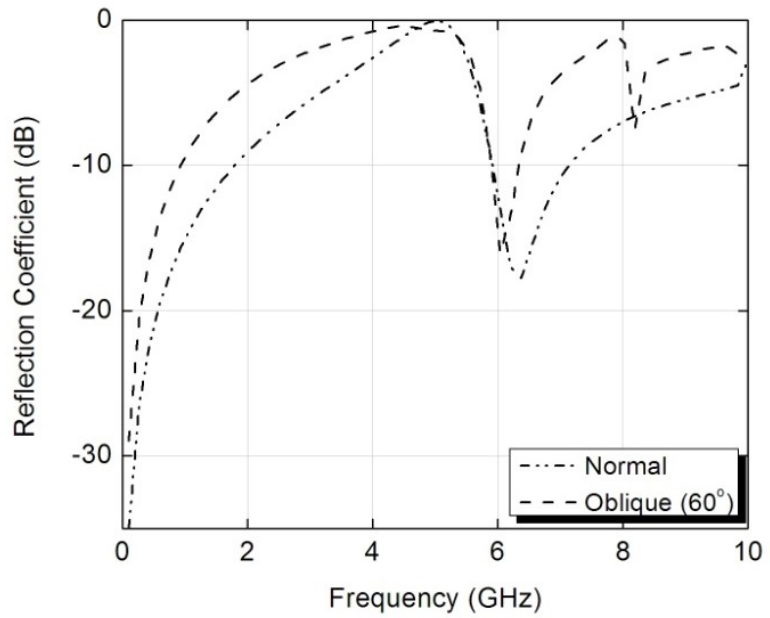

Fig. 5. Comparison Validation of the model for oblique $\left(60^{\circ}\right)$ incidence of a TE wave.

\section{TAMI ANTENNA DESIGN}

Circular patch antennas are usually used for wideband applications, and its performance is due to the radius of the conductor, the width, and length of feed line.

The planar antenna topologies analyzed and proposed in this work, are shown in Figures 6 and 7. They are fabricated of conductive patches with circular shapes, laid up on dielectric substrates, which are supported on ground planes.

The antennas were designed on Rogers RO4003 substrate with $\varepsilon r=3.55$ and thickness of $0.81 \mathrm{~mm}$. The resonant frequency was taken as $5.2 \mathrm{GHz}$ (IEEE $802.11 \mathrm{a}$ approach) and 
the radius of the circular patch was calculated by using (6) and (7) for the first approximation [9]. The antennas were optimized by the CST Studio design tool, software based on Finite Integration Technique (FIT).

$$
\begin{gathered}
a=F\left\{1+\frac{2 h}{\pi F \varepsilon_{r}}\left[\ln \left(\frac{\pi F}{2 h}\right)+1.7726\right]\right\}^{\frac{-1}{2}} \\
F=\frac{8.791 \cdot 10^{9}}{f_{r} \sqrt{\varepsilon_{r}}}
\end{gathered}
$$

where: a is the radius of the circular patch in $\mathrm{cm}, f_{\mathrm{r}}$ is the resonant frequency in $\mathrm{Hz}, \mathrm{h}$ is the substrate height in $\mathrm{cm}$, and $\varepsilon_{\mathrm{r}}$ the dielectric constant of the substrate.

The TAMI antenna was proposed using the tripolar array unitary cell, previously described in [11], and explored in previous section. The dimensions can be observed in Figure 7(b). Due to its symmetry, which to any incident wave provides an isotropic response, a better bandwidth and resonance is expected in comparison to a conventional circular UWB antenna, used as reference, with dimensions are shown in Fig. 6.

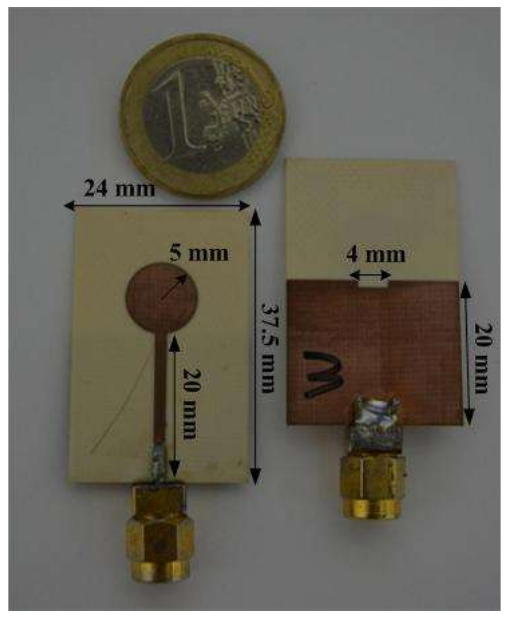

Fig. 6. Classical circular UWB antenna.

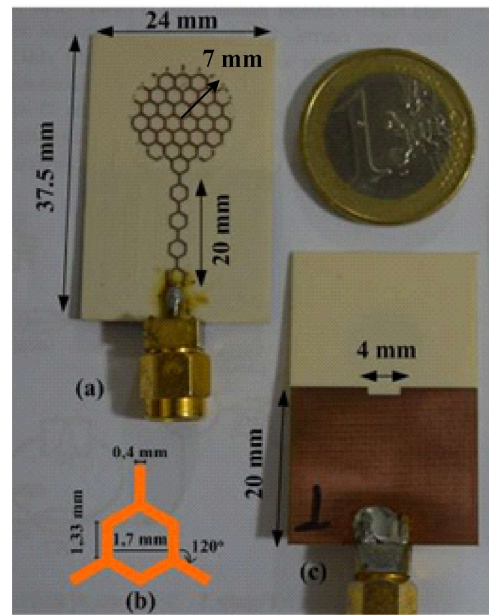

Fig. 7. Proposed TAMI antenna. (a) top view; (b) tripolar array dimensions; (c) ground plane.

\section{ANALYSis of Results}

In Figure 8, the input return loss (S11) for both antennas is shown. The impedance matching is analyzed by S11, which may be lower or equal to $-10 \mathrm{~dB}$ in the operation band, due to $90 \%$ of the antenna input power to be radiated in this case [22].

It can be observed the bandwidth increased around $32 \%$ on the range between $2 \mathrm{GHz}$ to $10 \mathrm{GHz}$. In Figure 9, the 2D normalized radiation pattern characteristics are presented. Two cuts are represented, where both of them have shown good performance of the antennas, with TAMI antenna keeping the same characteristics of the UWB antenna.

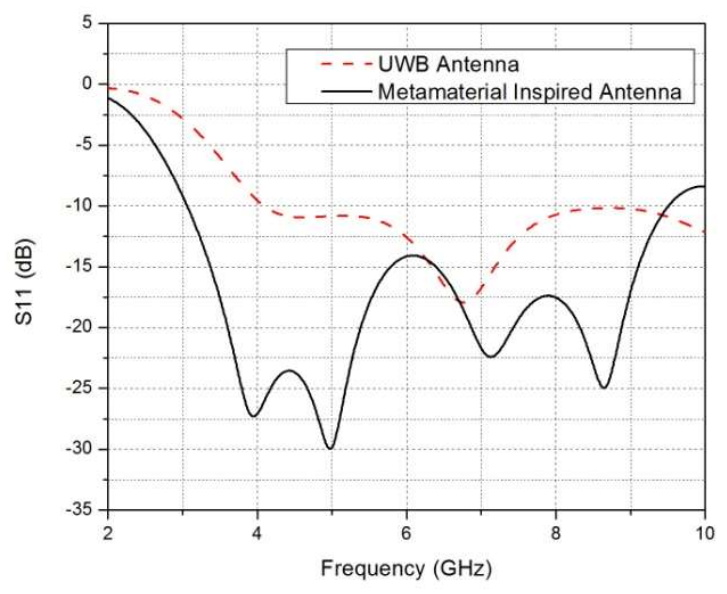

Fig. 8. Measured input return loss comparison for the UWB antenna and the proposed TAMI antenna.

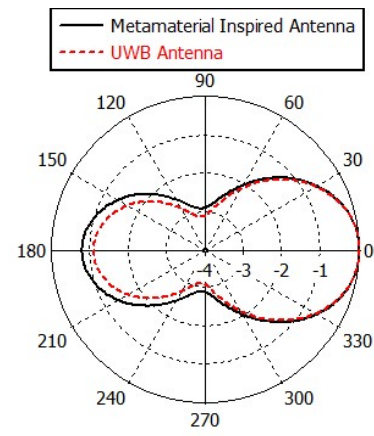

(a) theta/phi $=90^{\circ}$

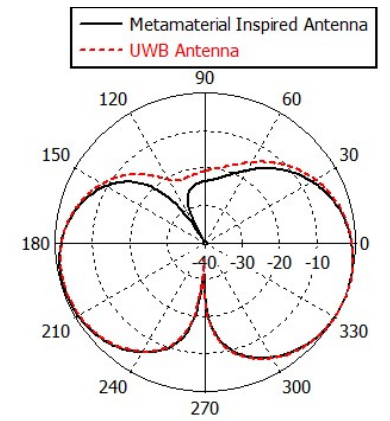

(b) phi/theta $=90^{\circ}$

Fig. 9. 2D Radiation pattern of TAMI antenna at $5.2 \mathrm{GHz}$.

In Figure 10, the 3D radiation pattern is presented. It can be noted that the resulting one looks kind of like a homogeneous semi-sphere radiating. The strongest energy is radiated outward, perpendicular to the TAMI antenna in the x-z plane. Notice that the 3D pattern meets a quasi-Omni directional behaviour, which evidences and ensures its good performance as a radiator element for applications at C-Band.

In addition, the obtained gain for the TAMI antenna shown an increase of $9 \%$ when it compares to the conventional UWB antenna. 
Figure 11 presents the surface current along the TAMI antenna. Due to its isotropic response to any incident wave, the current is symmetrically distributed on the device. From this property is possible to achieve best resonance, bandwidth, and gain during the process of antenna optimization, when compared to a conventional circular antenna.

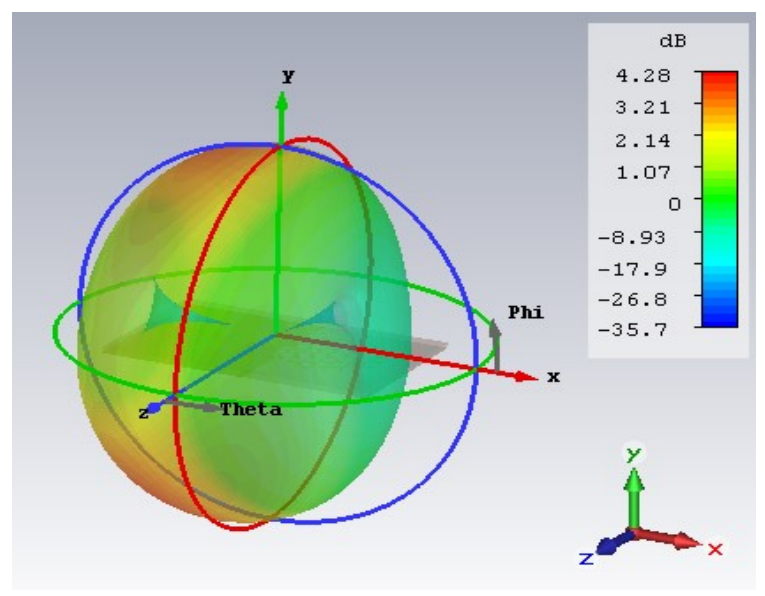

Fig. 10. 3D radiation pattern of TAMI antenna at $5.2 \mathrm{GHz}$.

Finally, the obtained results for the proposed TAMI antenna in comparison to the UWB antenna are summarized in Table 1 .

TABLE I

PLANAR ANTENNAS PROPERTIES COMPARISON

\begin{tabular}{cccc}
\hline \hline Topology & $\begin{array}{c}\text { Band } \\
\text { width } \\
\text { (GHz) }\end{array}$ & $\begin{array}{c}\text { Directivity } \\
\text { (dBi) } \\
\mathbf{a 5 . 2 G H z}\end{array}$ & $\begin{array}{c}\text { Gain } \\
\text { (dB) } \\
\text { @5.2GHz }\end{array}$ \\
\hline$U W B$ & 4.77 & 4.24 & 3.66 \\
TAMI & 7.01 & 4.24 & 4.28 \\
\hline \hline
\end{tabular}

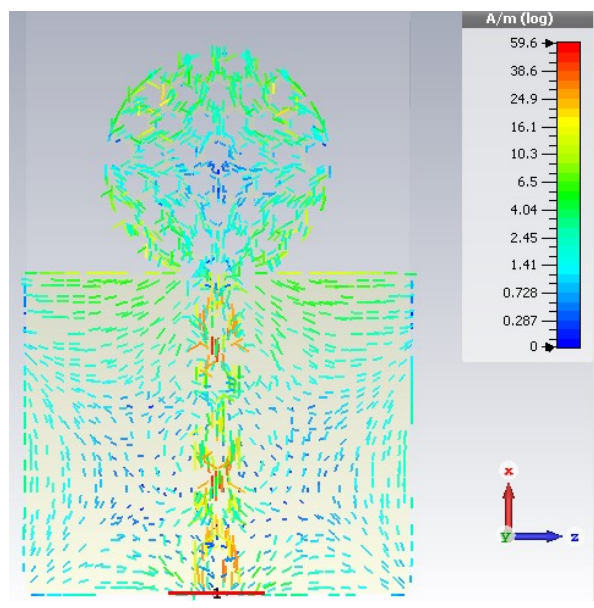

Fig. 11. Surface current along the TAMI antenna at $5.2 \mathrm{GHz}$.

\section{CONCLUSIONS}

In this paper, a C-Band Planar Metamaterial-Inspired Antenna was designed, fabricated, and characterized. The tripolar array metamaterial unitary cell was employed to improve the general response of the planar antenna. The obtained results were compared to the circular UWB antenna with the same structural and radiation characteristics.

The proposed antenna showed better results for resonance, bandwidth, and gain. Through experimental and simulated results, it can be observed an increase around 32\% on the bandwidth and $9 \%$ on the gain with the same radiation pattern characteristics. Thus, the TAMI antenna appears as a good option to attend broadband applications in C-Band for compact devices, including the 802.11a WLAN and radar applications.

\section{ACKNOWLEDGMENTS}

The development of this research was partially supported by the Federal University of Tocantins - Institutional Productivity Research Program (PROPESQ/UFT), and Santander Ibero-American scholarship program for Professors and Researchers. The authors also would like to thank Rogers Corporation for the samples.

\section{REFERENCES}

[1] Y. Zhao, Z. Zhang, and Z. Feng, "An electrically large metallic cavity antenna with circular polarization for satellite applications," IEEE Antennas Wireless Propag. Lett., vol. 10, pp. 1461-1464, 2011.

[2] M. S. R. Palacios and M. J. M. Silva, "Air substrate patch and monopole antennas in compact array for MIMO applications," in Proc. IEEE 7th Int. Conf. Elect. Eng. Comput. Sci. Automat. Control, Tuxtla Gutierrez, Mexico, Sep. 2010, pp. 305-308.

[3] Moh. Amanta K. S. Lubis, Fitri Yuli Zulkifli, Eko Tjipto Rahardj, "Design of Multiband Microstrip Antenna For Industrial, Scientific, and Medical Band Application", 2016 International Symposium on Electronics and Smart Devices (ISESD) November 29-30, 2016.

[4] Eko Tjipto Rahardjo, Fitri Yuli Zulkifli and Desi Marlena, "Multiband Microstrip antenna array for WiMAX application," in 2008 Asia-Pacific Microwave Conference, 2008, pp.1-4.

[5] [5] B. Badamchi, J. Nourinia, Ch. Ghobadi, and A. Valizade, "A Design of Compact Reconfigurable UWB Slot Antenna with Switchable Single/Dual band notch Function", IET Microwave and Antennas Propagation, Vol. 8, Iss. 8, pp. $541-548,2014$

[6] [6] R. Garg, P. Bhartia, I.Bahl, A.Ittipiboon. Microstrip Antenna Design Handbook. Norwood: Artech House, Inc., 2001.

[7] M.M Abd-Elrazzak, "Analysis and design of wideband planar yagi-uda and bi-yagi arrays with photonic band gap". Progress in Eletromagnetics Reserch C, Vol.19, 15-24, 2011.

[8] Le Chang, Zhijun Zhang, Yue Li, and Zhenghe Feng. "Air Substrate 2D Planar Cavity Antenna With Chessboard Structure", IEEE Antennas and Wireless Propagation Letters, vol. 16, pp. 321-324, 2017.

[9] A. C. Balanis. Antenna Theory - analysis and design. 3rd ed, John Wiley \& Sons, Inc., pp.811- 882, Hoboken, New Jerey, 2005.

[10] [10] Nam-I Jo, Dang-Oh Kim, and Che-Young Kim, "A Compact Band Notched UWB Antenna for Mobile Applications" PIERS ONLINE, Vol. 6, n. $2,2010$.

[11] E. F. Guelber, M. W. B. Silva, C. E. Capovilla, and H. X. Araujo . The design and application of the tripole array metamaterial unitary cell. Microwave and Optical Technology Letters, v. 58, p. 1786-1790, 2016.

[12] G. L. Ramos, C. E. Capovilla, H. X. Araujo, C. G. Rego, I. R.S. Casella, and P. T. Pereira, " A novel FDTD/WP-PML/NUFFT algorithm applied to the design of a printed metamaterial enhanced antenna", International Journal of Electronics and Communications (AEÜ), Vol. 70, 1187-1191, 2016.

[13] F. Capolino, Metamaterials Handbook: Applications of Metamaterials, CRC Press, pp. 20-1-21-12, 2009. 
[14] M. H. Rabah, D. Seetharamdoo, M. Berbineau, A. Lustrac. "New Metrics for Artificial Magnetism From Metal-Dielectric Metamaterial Based on the Theory of Characteristic Modes", IEEE Antennas and Wireless Propagation Letters, vol. 15, pp. 460 - 463, 2015.

[15] M. K. Karkkainen and P. M. T. Ikonen, "Finite-difference time-domain modeling of frequency selective surfaces using impedance sheet conditions," in IEEE Transactions on Antennas and Propagation, vol. 53, no. 9, pp. 2928-2937, Sept. 2005.

[16] R. Mittra, C. H. Chan, and T. Cwik, "Techniques for analyzing frequency selective surfaces-A review," Proc. IEEE, vol. 76, pp. 15931615, Dec. 1988.

[17] C. K. Lee and R. J. Langley, "Equivalente circuit models for frequency selective surfaces at oblique angles of incidence", IEE Proceedings, 132 (6), $395-399$ (1985).

[18] R. Langley and A. Drinkwater, "Improved empirical model for the jerusalem cross," Microwaves, Optics and Antennas, IEE Proceedings on, vol. 129, no. 1, pp. 1-6, Feb. 1982.

[19] F. Costa, A. Monorchio and G. Manara, "Efficient Analysis of Frequency-Selective Surfaces by a Simple Equivalent-Circuit Model," in IEEE Antennas and Propagation Magazine, vol. 54, no. 4, pp. 35-48, Aug. 2012.

[20] M. W. B. Silva, A. L. P. S. Campos, and , L. C. Kretly " Design of thin microwave absorbers using lossy frequency selective surfaces". Microwave and Optical Technology Letters, 57: 928-933, 2015.

[21] O. Luukkonen, C. Simovski, G. Granet, G. Goussetis, D. Lioubtchenko, A. V. R"ais"anen, and S. A. Tretyakov, "Simple and accurate analytical model of planar grids and high-impedance surfaces comprising metal strips or patches," IEEE Transactions on Antennas and Propagation, vol. 56, no. 6, pp. 1624-1632, 2008.

[22] L.C. Kretly, C.E. Capovilla, "Analysis of radiation patterns and broadband characteristics of a novel quasi-yagi antenna array for wireless communications" IEEE International Symposium on Wireless Personal Multimedia Communications, Japan, 2003.

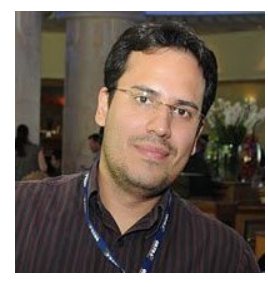

Humberto X. Araujo received his Ph.D. from University of Campinas in 2012. His field of interest includes Electromagnetic Compatibility, metamaterials, analog integrated circuits, active microwave devices and antennas. He has expertise on the design and development of setups for EMI/EMS measurements, and metamaterials prototypes. Nowadays, he is with the Federal University of Tocantins, as Professor of the Electrical Engineering.

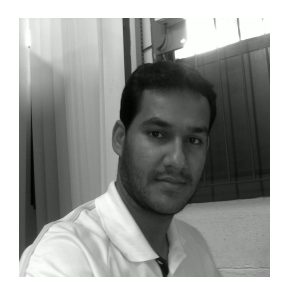

Mauricio W. B. Silva wasborn in Belém City, Brazil in 1980. He received his M.Sc. and Ph.D. degrees in electrical engineering from the University of Campinas, in 2009 and 2014 respectively. His field of research currently involves MEMS, Metamaterials, antennas, and microwave devices. From 2015 to 2016, he was a postdoctoral research with the Federal Fluminense University. He is currently Professor with the Federal University of Roraima.

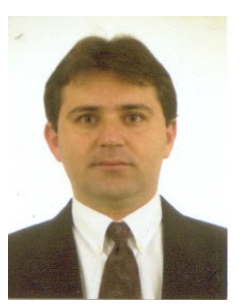

Ivan Roberto Santana Casella received his M.S. and $\mathrm{PhD}$. in Electrical Engineering from the Polytechnic School of the University of Sao Paulo (EPUSP). Part of his $\mathrm{PhD}$ was developed at the University of Toronto (UofT) in Canada, where he acted as researcher and teacher assistant. He worked as electronic designer at the Consumer Electronics Development Laboratories of Philips and as researcher at the Automation and Informatics Research and Development Center of NEC (CPDIA) in the design and analysis of analog and digital systems. He is now Adjunct Professor IV at the Federal University of $A B C$ (UFABC) and chair of the Communication and Information Laboratory (LIC). His current interests are in the areas of wireless communication and smart grid.

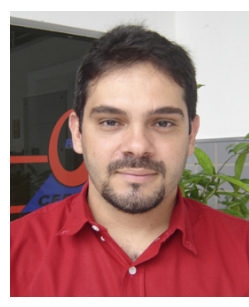

Antonio L. P. S. Campos received the B.S.E.E. and M.S.E.E. degrees from the Federal University of Rio Grande do Norte, Natal RN, Brazil, in 1996 and 1999, respectively, and he received the Ph.D. degree from Federal University of Paraíba, Campina Grande PB, Brazil. Since 2010, he has been with the Federal University of Rio Grande do Norte, Natal RN, Brazil, where he is currently a Professor of the Communication Engineering Department. He is currently involved in the areas of FSS, scattering of electromagnetic waves, antennas, and planar devices. He is a member of the Brazilian Microwave and Optoelectronics Society (SBMO).

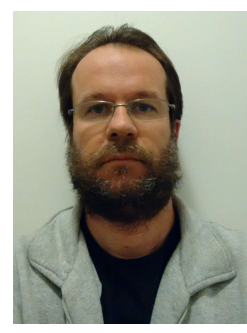

Carlos Eduardo Capovilla was born in Vinhedo, Brazil, on March 06, 1977. He received the B.S. degree from the University of Sao Paulo in 2001, M.Sc. and Ph.D. degrees from the University of Campinas in 2004 and 2008, all in Electrical Engineering - RF Microelectronics. $\mathrm{He}$ is currently a Professor with the Federal University of $\mathrm{ABC}$ (UFABC). His current research interests include RF CMOS integrated circuits, smart antennas, mobile systems design, smart grid applications, and telecommunications in complex 